\title{
Genetical and Biochemical Aspects of Anthocyan Pigments
}

\section{By Muriel Wheldale Onslow}

$\mathrm{S}^{\mathrm{I}}$ NCE the publications of Willstätter and his co-workers upon anthocyan pigments during the years 1913-16, other investigators have turned their attention to this field of research. Though the later work has scarcely introduced any fresh facts of fundamental importance about this group of pigments beyond those already presented by Willstätter himself, yet it has given us much additional knowledge as to the distribution of the individual pigments among the flowering plants, especially in connexion with colour varieties of various species. The object of this article is to indicate how the chemical identification of the pigments may be correlated with certain broad aspects of the genetics of flower colour and the metabolic reactions connected with formation of anthocyan pigments in the plant itself.

From Willstätter's investigations the fact emerges that there are three commonly distributed anthocyan pigments-pelargonidin, cyanidin, and delphinidin :
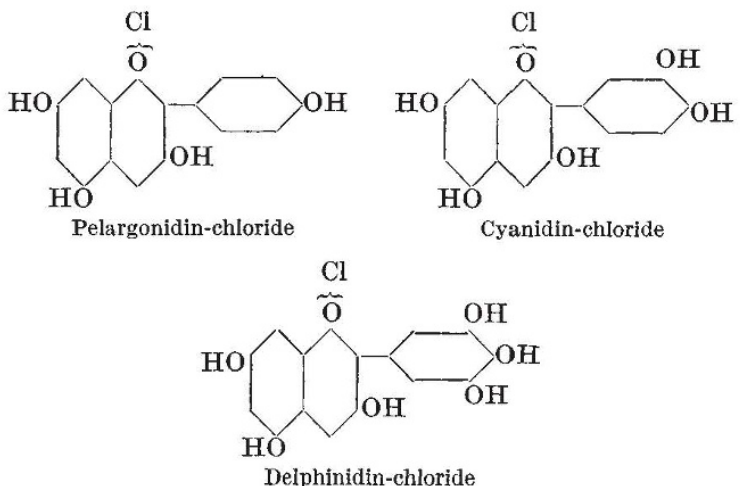

Cyanidin and, especially, delphinidin appear also, according to Willstätter, in various plants as methyl ethers, certain hydroxyl groups being replaced by methoxyl groups. The positions, however, of the methoxyl groups were not defined precisely. Moreover, though the sugars of the glycosidal pigments were identified, the points of attachment of the sugar groups were not definitely ascertained.

The more recent investigations ${ }^{1}$ include those of Karrer in Zurich and Robinson in Great Britain. Karrer and his co-workers have determined the positions of the methoxyl groups in such pigments as are methylated; also to some extent those of the sugars. Robinson and his co-workers have synthesised a number of benzopyrylium compounds, some of which have been identical with the natural pigments, and thus they have confirmed certain of Willstätter's conclusions. They have defined in many cases the position of the sugars and have synthesised some naturally occurring glucosides. Moreover, by elaboration of tests, they have made it possible to identify with relative ease various known anthocyan pigments in any given material.

No. 3260 , VoL. 129]
For the purposes of this article, the presence of methoxyl groups and the kind and the position of the sugars are of subsidiary importance. The points at issue are concerned chiefly with the occurrence of the three main pigments.

Let us turn first to genetical problems. In the majority of horticultural plants which have been long under cultivation it is difficult to determine which species have been concerned in their origin. In a few cases, such as the sweet pea, Chinese primula, and snapdragon, it is relatively simple.

In the snapdragon (Antirrhinum majus) the pigment of the magenta flowers is cyanidin, and there is no doubt that this is the pigment of the original, wild, magenta-flowered type. About thirty years ago the most important of recent 'breaks' in Antirrhinum appeared-rose doré-a variety now catalogued under many names, such as coral, salmon pink, etc. Genetically, rose doré is hypostatic (recessive) to magenta, and there is a factorial difference. Though not yet isolated, there is no doubt that the flowers of rose dore contain pelargonidin. The stems, petioles, and leaves often develop pigment too; in the original magenta type it is cyanidin, in the variety, pelargonidin. Here, then, we have the creation from a plant producing cyanidin of another which produces pelargonidin instead. Moreover, it should be realised that the power to produce one or the other pigment may be limited to the epidermal layer of the plant. In some horticultural types and varieties two or more pigments may be present together, though one usually predominates. Such complications are eliminated from the present discussion, especially as the segregation of pigments is usually complete.

Now a similar case to that of Antirrhinum has long been obvious from Willstätter's original results. He found the blue cornflower (Centaurea Cyanus) to contain cyanidin; its pink variety, pelargonidin. Though the genetics of the cornflower may not have been demonstrated in a practical way, it is clear to a geneticist that there is a factorial difference between blue and pink, and that pink is hypostatic.

Another case is the blue flower of Hyacinthus orientalis, which contains delphinidin. Though the origin of many horticultural bulb plants is doubtful and has often been kept secret, one must suppose the colour of the original $H$. orientalis to have been blue. The pink variety contains, again, pelargonidin, and cyanidin is claimed for intermediate varieties. In Nature, too, a pink variety of the wild hyacinth (Scilla nutans) may be found occasionally. In the latter we have undoubtedly a phenomenon of variation alone. In blue Campanula delphinidin is reported, and we cannot doubt that the original type was blue. Again, a hypostatic pink variety exists and contains pelargonidin. Thus from a delphinidin type also have arisen varieties containing pelargonidin and, sometimes, cyanidin. This true red (pelargonidin)

\footnotetext{
○ 1932 Nature Publishing Group
} 
group of the geneticist, hypostatic to cyanidin and delphinidin groups, is obviously present in a number of cultivated plants, such as the sweetwilliam, Chinese primula, stock, phlox, carnation, sweet pea, and Clarkia. To determine when it is a true sport (Antirrhinum, Chinese primula, and sweet pea) and when it may result from species crossing is, however, difficult.

Now the interesting question arises whether, conversely, starting with pelargonidin, the power to form cyanidin may arise by variation; or, starting with cyanidin, the power to form delphinidin (all pigments again may be limited to the epidermis). Evidence in support of this supposition is difficult to acquire. The varieties of types under long-standing cultivation may show, among them, all three pigments, but since there is often uncertainty as to the outward appearance of the original type, so there is also uncertainty as to its pigmentation. Thus in the sweet pea, of which the original type resembled 'Purple Invincible', and in the Chinese primula, where the original type is known, we have not yet been informed as to their pigmentation. Such types may have contained either delphinidin or cyanidin, or both. In the aster, chrysanthemum, gladiolus, gloxinia, phlox, tulip, and others the problem is even more difficult. There is, however, a case where it would appear that a pelargonidin type has given rise to a cyanidin variety. Such is the garden nasturtium (Tropceolum majus). The orange-red type contains pelargonidin; purple-red varieties, which are hypostatic to the type, contain cyanidin.

Thus, summing up, we may say that loss of one series of factors leads to loss of power of oxidation in the phenyl ring, so that instead of delphinidin, cyanidin is formed, and instead of cyanidin, pelargonidin. There is little doubt, too, that the reverse effect takes place. This we may express as the loss of a series of factors which inhibit oxidation; in other words, loss of another series of factors leads to increased oxidation. So that, instead of pelargonidin, cyanidin may be formed, and instead of cyanidin, delphinidin.

The occurrence of a more oxidised pigment in a hypostatic variety is also to be found in the inheritarice of flavone pigments in Antirrhinum. Two varieties, ivory and yellow, exist, and of these ivory contains apigenin, yellow, luteolin :
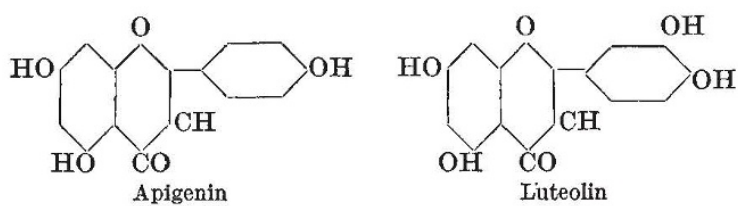

There is a factorial difference between the varieties, and ivory, moreover, is epistatic (dominant) to yellow.

Now the question arises as to what may be the significance of the facts so far quoted. I have already pointed out ${ }^{2}$ that evidence in general indicates the origin of anthocyan pigments from condensation of residues of amino-acids after deamination.
There are three classes of aromatic compounds which may be synthesised from such residues, the flavone pigments, * the anthocyan pigments, and the tannins. Flavone pigments are universally distributed in the tissues under all conditions. Hence it is likely that they are products of de-amination and condensation under the usual conditions of growth and development. Anthocyan pigments are only formed under special conditions. Finally, the tannins only arise in a certain type of plant.

Upon the precise reactions of condensation lead. ing to the formation of anthocyan pigments it is idle to speculate. The phenyl ring, we would assume, has its origin from tyrosine or phenylalanine. The phloroglucinol ring, possibly, from short-chained residues of aliphatic amino-acids; or again, possibly, from hexose. By oxidation, either by enzymes or chemical agents, hydroxyl groups are readily introduced into the phenyl ring in a position ortho to that of an existing group. Clearly, the occurrence and segregation of pelargonidin, cyanidin, and delphinidin is a matter of loss or gain of power of oxidation or reduction.

It is obvious from the foregoing that the wide range of coloration in flowers is to some extent due to the pigment involved. Summarising on very general lines, one may say that pelargonidin flowers (though these are rare in natural species) incline to shades of scarlet; cyanidin flowers to shades of crimson or purple-red; delphinidin flowers to shades of blue or purplish-blue. But there are many exceptions to such a classification. The scarlet poppy, the magenta rose, and the blue cornflower contain the same pigment, cyanidin. In the Chinese primula, two varieties, blue and magenta, exist having the same pigment.

The idea has prevailed for a century or more that the colour of a flower may be changed by the presence of various substances in the petals, such as acids, alkalis, iron salts, and other compounds. Willstätter showed that the blue colour of the cornflower is due to a potassium salt of cyanin. He also constantly emphasised his view that the colour of the flower or fruit may be modified by accompanying substances in the tissues. Recently, Robinson ${ }^{3}$ has brought forward the idea of the existence of copigments in many cases, that is, a variety of substances occurring in the plant which, entering into loose combination with the anthocyan pigments, modify their colour. Such a substance may represent a factorial difference between varieties.

So much for variation in colour. A suggestion can also be made here in connexion with the cause of albinism. In Antirrhinum there are two forms of albino in regard to anthocyan pigments, namely, ivory (including the hypostatic yellow) and white. The ivory contains flavone pigment ; the white has none. Ivory and white (of correct constitution) crossed together will produce the magenta type. That is, ability to form flavone pigment is one essential for anthocyanin production; the other is a factor borne by white. Such white-flowered plants are always stunted and poorly developed.

* The expression flavone pigment is collective and includes both flavone and flavonol. 
They contain no flavone pigment, and hence can never form anthocyan pigment; though not necessarily derived from the yellow pigment, anthocyanin production depends on its presence.

It is reasonable to suggest that some aromatic component of the protein is entirely absent from the white-flowered plants. Hence neither anthocyan nor flavone pigment can be formed. A different cause for albinism must be postulated for the ivory variety, though there is no indication at present as to what this may be. Two kinds of albino, containing between them the elements essential for the production of anthocyan pigment, occur in the sweet pea and many other plants, but in these cases they are usually indistinguishable to the eye.

There is yet another form of variation among cultivated plants of rather a different nature from those we have already considered. In this the type has flowers, either free from anthocyan pigment or only slightly tinged, whereas the variety is fully coloured. That is, loss of a factor gives rise to a fully pigmented, hypostatic variety. The origin of the red variety of the sunflower (Helianthus annuus) from the yellow type affords an example; so also does the purple-leaved (copper) beech.

Just such a phenomenon as the above mentioned, that is, the appearance or non-appearance of anthocyanin, is characteristic of the flowering plants as a whole. The flowers of certain species form very little or no anthocyan pigment, while it is abundant in the flowers of others. It is difficult to make any suggestion as to what may be the chemical interpretation. It is conceivable that presence of sugar in the tissues may prevent the de-amination in inhibited types; or that excess of sugar may bring about condensation, as is probably the case when anthocyanin develops as the result of artificial sugar feeding. If so, then the factors concerned are correlated with some metabolic cycle other than that of pigmentation.

Before we leave the above variation among species one more point may be made, namely, the resemblance of the phenomenon to the distribution of autumnal anthocyan pigment in leaves, for the latter also is present in some species and absent from others. In fact, the autumnal coloration of leaves may be regarded as quite analogous to the pigmentation of petals. In each case the organ is senescent; it has ceased to assimilate and is gradually becoming detached from the parent plant. Desiccation is enhanced in the petal by the unprotected condition of the tissues against loss of water; in the autumnal leaf by the decreased water absorption from the soil owing to low temperature. An autumnal leaf developing anthocyan pigment can be regarded as a petal of very coarse texture. When anthocyan pigments are not developed it is yellow, as is also a petal which owes its colour to plastid pigment and has not the power to form anthocyan pigment.

Finally, some additional points of interest are connected with a consideration of the relationship of the particular anthocyan pigment formed in a plant to the accompanying flavone pigments and other aromatic compounds in the same plant. If we ascertain the relative frequency of occurrence of cyanidin and delphinidin among the plants investigated, we find the values to be approximately as follows:

\begin{tabular}{|c|c|c|}
\hline & $\begin{array}{c}\text { Plants contain- } \\
\text { ing cyanidin. }\end{array}$ & $\begin{array}{c}\text { Plants contain- } \\
\text { ing delphinidin. }\end{array}$ \\
\cline { 2 - 4 } $\begin{array}{c}\text { Where isolation of pig- } \\
\text { ment has been achieved }\end{array}$ & 12 & 12 \\
$\begin{array}{c}\text { Where pigment is identi- } \\
\text { fied by reactions only }\end{array}$ & 61 & 60 \\
\hline
\end{tabular}

Thus plants appear to form an anthocyan pigment having the three hydroxyl groups characteristic of gallic acid as frequently as one with the dihydroxy grouping characteristic of catechol.

Now an outstanding fact of importance in connexion with aromatic compounds in the flowering plants is the distribution, in about 63 per cent of the natural orders, of the catechol oxidase system involving the presence of substances containing the dihydroxy grouping of catechol. From the remaining plants catechol derivatives are absent, pyrogallol compounds being frequently formed instead.

For all cases available I have made a record of the type of anthocyanin formed and the presence or absence of the catechol oxidase in the same plant. It is clear from the outset that the presence of the latter cannot always be associated with formation of cyanidin and its absence with formation of delphinidin, since the frequency of occurrence of cyanidin to that of delphinidin is $1: 1$, while that of oxidase to non-oxidase plants is almost $2: 1$. Such a conclusion is also apparent on analysis of the cases of species producing delphinidin; instead of being universally without oxidase, some contain oxidase, while others are non-oxidase plants. Hence on broad lines there is apparently no indication that plants producing catechol compounds always produce cyanidin.

Nor can any consistent relationship be detected between the anthocyan and flavone pigments of individual plants. Thus, for example, both flavone and flavonol pigments occur in plants :

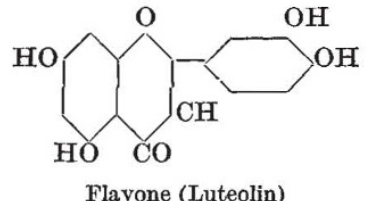

Flavone (Luteolin)

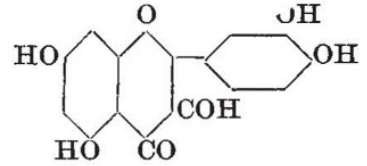

Flavonol (Quercetin)
But all natural anthocyan pigments, on the simple reduction relationship, are related to flavonols, none to flavones.

In Rosa gallica and the wallflower there is a certain symmetry in the relationship of flavone to anthocyan pigment, the former being quercetin, the latter cyanidin; yet in other cases, such as Delphinium (kæmpferol, delphinidin), Viola (quercetin, delphinidin), and Antirrhinum, there is no such connexion.

$$
\text { No. 3260, VoL. 129] }
$$


In varieties of Antirrhinum the four pigments, cyanidin, pelargonidin, apigenin, and luteolin, are formed. But there is no correlation between their presence in the epidermis. Cyanidin or pelargonidin can be found either with apigenin or luteolin.

General considerations would suggest that possibly in the flower flavone pigments result from de-amination connected with growth and development; anthocyan pigments with de-amination connected with senescence.

1 The investigations of Karrer and his co-workers are published in Helv. Chim. Acta; those of Robinson and his co-workers in $J$. Chem Soc. and Biochem. $J$. Details of the data on genetics can be found in the $J$. Genetics and Onslow's "Anthocyanin Pigments of Plants".

2 Nature, 128, 373, Aug. 29, 1931.

Biochem. J., p. 1687, 1931 .

\section{The Eruptions in the Andes \\ By Dr. Charles Davison}

$\mathrm{E}^{A}$ ARLY in the morning of April 10, eruptions began in several volcanoes of the southern Andes, most of which were supposed to be dormant or but rarely in action. The volcanoes affected, from north to south, are Tupungato, Overo, Tinguiririca, Peteroa, Descabezado, Las Yeguas, and Quizapu. Their heights diminish southwards, from $21,810 \mathrm{ft}$. for Tupungato to $11,342 \mathrm{ft}$. for Las Yeguas. All of them were in action simultaneously, though the distance that separates the extreme mountains is about 200 miles.

The principal feature of the eruptions is the great amount of the solid materials ejected. Loud explosions were heard at Santiago on the west side, and throughout the department of San Rafael on the east side, one hundred miles or more from the nearest volcano. But they were evidently not to be compared with those during the eruption of Krakatau in 1883, the sounds of which were heard at several places more than 2000 miles from the island, and at one place 2968 miles distant. Some observers in an aeroplane crossed the great crater of Las Yeguas (apparently on April 12). Every half-minute, loud explosions occurred within it, and blocks weighing many tons were thrown upwards more than $200 \mathrm{ft}$. These, it was said, gave the impression of being pulverised in mid-air.

During the three days of activity - the eruptions had almost ceased on April 13-all the towns near the volcanic zone were in a state of semi-darkness owing to the steady fall of fine dust and ashes. On the west side, the country from Santiago to Talca was covered with a layer of whitish dust, in places more than two inches deep. On the east side, the fall was much heavier. In the department of San Rafael, the layer of dust was a foot in depth, in other places more than two feet, and in one nearly three feet, so that trains were stopped from running. Even at Montevideo, about 850 miles from the nearest volcano, there was a steady fall of dust for many hours. Over Buenos Ayres (730 miles), it is estimated that more than 3000 tons of debris have fallen. The whole country there is coated with grey dust, so that cattle have to be fed with hay and artificial foods. Even if the average thickness of the dust over the whole area of deposition were no more than one-tenth of an inch, the total volume of the fallen dust would be about five cubic miles.

Several villages in the province of Mendoza were shaken by earthquakes, the stronger, no doubt, among many hundreds felt in the neighbourhood of the volcanoes. The shocks that accompany a volcanic eruption, though sometimes destructive within a small area, are seldom felt more than a few miles from their origins. Occasionally, as in Hawaii in 1868 and Sakura-jima in 1914, a tectonic earthquake strong enough to be registered all over the world may occur in the immediate neighbourhood of the volcano, but such disturbances are rare.

About one hundred years ago, on Feb. 20, 1835, an earthquake of somewhat similar type overthrew Concepcion, and with it a long stretch of the Chilean coast was uplifted, in one place by $10 \mathrm{ft}$. One month before this, as Darwin describes in his great memoir, "On the Connexion of Certain Volcanic Phenomena in South America . . .", eruptions occurred in three volcanoes, Osorno, Aconcagua, and Coseguina, all beginning within six hours. The first two mountains are separated by 550 miles. The third is so distant from either that, as Darwin says, we cannot be too cautious in assuming that the phenomena were connected. At the moment of the Concepcion earthquake, the volcanoes of Osorno and Minchinmadom, 150 miles apart, burst into action. Some months later, on Nov. 11 of the same year, a severe earthquake occurred at Talcahuano, the port of Concepcion, and on the same day, Osorno and Corcovado, separated by 205 miles, burst into violent action.

To us at a distance, a question of great interest is whether the eruptions will be followed in due time by such wonderful sunsets as were observed all over the world for months after the Krakatau. eruption of 1883 . With regard to the amount of dust projected into the atmosphere, there can be little doubt. The question is whether enough could rise to heights of from eight to ten miles. As Capt. Cave remarks in a letter to the Times (April 14), the explosions seem to have been less violent than those of Krakatau. On the other hand, the height of Krakatau was 2623 feet, while the Andean volcanoes rise from two to three miles higher. On April 13, Capt. Ralph Wooten, the U.S. Air Attaché at Santiago de Chile, flew across Quizapu at an altitude of $14,000 \mathrm{ft}$. He estimated that the smoke column was then reaching $5000 \mathrm{ft}$. above the crater, but he considers that, on April 10, at the moment of greatest activity, it must have risen to a height of $30,000 \mathrm{ft}$. If this estimate is correct, or nearly so, it seems possible that, within a few months, sunset glows may be visible in Great Britain. The eruption of Krakatau, it will be remembered, occurred on Aug. 26-27. The sunset glows were not observed in England until the end of November.

* Trans. Geol. Soc., vol. 5, pp. 601-631; 1840. 